\title{
Microcomposants électromécaniques pour la réalisation de références de tension en courant alternatif
}

\author{
Micro-electromechanical components \\ for alternating voltage reference
}

\author{
Alexandre BOUNOUH ${ }^{1}$, François BLARD ${ }^{1,2}$, Henri CAMON $^{2,3}$, Denis BELIERES ${ }^{1}$ et François ZIADE $^{1}$ \\ ${ }^{1}$ LNE, 29 Avenue Roger Hennequin, 78197 Trappes Cedex, France, alexandre.bounouh@lne.fr. \\ 2 CNRS; LAAS, 7 Avenue du Colonel Roche, 31077 Toulouse Cedex 4, France. \\ 3 Université de Toulouse; UPS, INSA, INP, ISAE; LAAS ; 31077 Toulouse Cedex 4, France.
}

\begin{abstract}
Résumé
Ce travail explore les potentialités des microsystèmes électromécaniques (MEMS) pour fabriquer des références de tension en courant alternatif (AC) au moyen du couplage électromécanique pour des applications en métrologie et en instrumentation miniaturisée. Des structures de test de référence de tension $\mathrm{AC}$ de $2 \mathrm{~V}$ à $100 \mathrm{~V}$ ont été conçues et fabriquées en utilisant un procédé de micro-usinage de surface de couche épitaxiale de silicium d'un substrat silicium sur isolant (SOI). Ce procédé permet un contrôle précis à la fois des dimensions du système et des propriétés du matériau. Les valeurs mesurées des références de tension MEMS sont en bon accord avec les simulations effectuées avec le logiciel Coventor. Ces structures de test ont également servi à développer l'électronique de commande et d'actionnement des MEMS et les bancs de mesure et de caractérisation. Une deuxième génération de microsystèmes avec des caractéristiques améliorées a ensuite été fabriquée. Les fréquences de résonance mécanique de ces nouveaux MEMS mesurées par DLTS (Deep Level Transient Spectroscopy) sont de quelques kilohertz. Cela permet d'envisager le développement de références de tension $\mathrm{AC}$ fonctionnant à partir de quelques dizaines de kilohertz. Enfin, la stabilité de la tension de référence MEMS, évaluée à $100 \mathrm{kHz}$, est très prometteuse puisque pour les meilleurs échantillons, l'écart relatif par rapport à la valeur moyenne présente un écart type de $6,3 \times 10^{-6}$ sur près de $12 \mathrm{~h}$.
\end{abstract}

MOTS CLÉS : MÉTROLOGIE ÉLECTRIQUE, MEMS, RÉFÉRENCE DE TENSION, SOI.

\footnotetext{
Abstract

This work explores MEMS (Micro-ElectroMechanical Systems) potentialities to produce flexible AC voltage references through mechanical-electrical transduction that could be used for high precision electrical metrology and for applications in miniaturized
}

instrumentation. AC voltage references ranging from $2 \mathrm{~V}$ to $90 \mathrm{~V}$ have been designed and fabricated using the same Epitaxial Silicon On Insulator (SOI) Surface Micromachining process that permits an accurate control of both dimensions and material properties. The measured MEMS AC voltage reference values have been found in a good agreement with the calculations performed with Coventor software. These test structures have been used to develop the read-out electronics to drive the MEMS and to design a second set of devices with improved characteristics. Deep Level Transient Spectroscopy measurements carried out on the new MEMS showed resonance frequencies of about a few kilohertz, which makes it possible to have AC voltage references working from about tens of $\mathrm{kHz}$. Moreover, the stability of the MEMS out-put voltage at $100 \mathrm{kHz}$ has been found very promising for the best samples where the relative deviation from the mean value over almost $12 \mathrm{~h}$ showed a standard deviation of $6.3 \times 10^{-6}$, which is a very good result.

KEY WORDS: ELECTRICAL METROLOGY, MEMS DESIGN, VOLTAGE REFERENCE, SOI PROCESS.

\section{Introduction}

La révolution des nanotechnologies et nanostructures n'a pas seulement permis de mettre en évidence des phénomènes physiques nouveaux liés à des effets quantiques de taille, de confinement, de surface et d'interface dans les matériaux, ce qui est d'ailleurs à l'origine du bouleversement qu'a connu la métrologie électrique à travers l'effet Hall quantique et l'effet Josephson, mais a aussi ouvert un nouveau champ d'applications technologiques dont la portée économique est considérable mais 
où la physique est encore loin d'avoir établi des bases définitives : il s'agit bien évidemment des microsystèmes électromécaniques (MEMS). Rappelons que les MEMS sont des systèmes microscopiques qui associent des éléments mécaniques, optiques, électromagnétiques, thermiques mais aussi fluidiques à de l'électronique sur des substrats semi-conducteurs. Ces systèmes s'élaborent à partir de techniques de lithographie, dépôt et gravure (micro-usinage de surface et de volume, et les technologies telles que LIGA qui signifie lithographie, électrodéposition par bain d'électrolytes et moulage) et assurent des fonctions de capteurs de paramètres physiques (pression, accélération...) ou d'actionneurs. Dans ces systèmes, le principe d'actionnement peut être basé sur des matériaux déposés dits «actifs » qui mettent ensuite en jeu les propriétés de piézoélectricité, de magnétostriction ou de mémoire de forme. Un autre type d'actionnement est lui basé sur une action externe qui consiste à appliquer un champ électrique pour générer un mouvement, mettant en jeu la force électrique de Coulomb qui apparaît entre deux électrodes portées à des potentiels différents. On peut aussi avoir un actionnement magnétique, thermique ou électrochimique. Cependant, les MEMS utilisant le principe d'actionnement électrostatique sont de loin les plus répandus pour leurs structures relativement simples et surtout parce qu'ils peuvent être fabriqués à partir de matériaux bien connus. Les atouts de la technologie MEMS résident dans l'amélioration de la performance des produits, l'ajout de nouvelles fonctionnalités, la réduction de la consommation d'énergie, la production en masse, la miniaturisation et l'accroissement de la fiabilité et de l'intégration.

L'essor de plus en plus important qu'ont connu les MEMS depuis les années 1970, avec les premiers capteurs de pression, vient de la capacité d'assemblage intelligent de dispositifs de base (poutres, ressorts, roues, engrenages...) pour fabriquer des microsystèmes complexes (moteurs, résonateurs, actionneurs, pompes...). C'est ainsi qu'aujourd'hui la production de masse des MEMS concerne des domaines aussi variés que la défense, le secteur médical, l'électronique, les télécommunications et l'automobile. De plus, les MEMS sont en train d'investir les domaines des télécommunications optiques, des radiofréquences et des biocapteurs devenant une technologie de choix pour ces secteurs et engendrant pour chacun d'entre eux des petites révolutions car ils permettent d'envisager la réalisation d'architectures présentant de nouvelles fonctionnalités aux performances améliorées. Dans beaucoup de cas, l'introduction des MEMS ne va pas se traduire uniquement par un remplacement des composants existants mais par un changement complet dans l'architecture du système lui-même. Cette émergence de l'industrialisation va se traduire par un besoin crucial de techniques de test appropriées à ces composants afin de mieux connaître leurs propriétés et ainsi de pouvoir concevoir des systèmes à base de MEMS avec une grande confiance.

Depuis une dizaine d'années, de nombreux travaux de recherche visent à développer des circuits à base de MEMS. L'instrumentation miniaturisée est un des champs d'application privilégiée and des MEMS car, par leurs dimensions et les différents couplages que l'on peut obtenir, il est possible de développer des composants tout à fait innovants. À partir de dispositifs MEMS constitués de membranes, de poutres ou de bascules, les premières applications en métrologie électrique ont vu le jour vers 1995 avec les premiers convertisseurs alternatif-continu (AC-DC) constitués d'une bascule dans laquelle on réalise l'équilibre entre les forces électriques engendrées par des tensions AC et DC [1,2]. Par la suite, plusieurs équipes de recherche ont montré d'autres possibilités d'applications des MEMS pour la métrologie électrique : références de tension AC et DC [3-6], convertisseurs ACDC $[7,8]$, référence de courant et diviseur de tension basse fréquence $[9,10]$, et capteurs de puissance haute fréquence [11-13].

La technologie MEMS permet d'envisager la réalisation de références secondaires de tension électrique de valeurs allant de quelques volts à quelques centaines de volts avec une grande stabilité. Les points forts des MEMS sont d'abord l'absence de contact mécanique et donc d'usure, ensuite l'effet d'amortissement de la membrane peut être contrôlé via le contrôle de la pression du gaz résiduel dans la capsule du dispositif, et enfin les caractéristiques des composants peuvent être déterminées par des calculs de mécanique classique. Cependant, pour les très hautes performances recherchées, il est indispensable de limiter la sensibilité de ces structures aux contraintes mécaniques et de mettre au point une électronique de contrôle adaptée et très performante qui tient compte, d'une part des valeurs très faibles des capacités de travail dans ces dispositifs, et d'autre part du phénomène du «pull-in », base des références de tension.

Les dispositifs MEMS utilisés en métrologie électrique sont formés d'électrodes micro-usinées dont au moins une est mobile, se déplaçant grâce à l'application d'une force électrostatique attractive entre les deux électrodes qui est inversement proportionnelle au carré de la distance entre les électrodes. La force de rappel de raideur caractéristique permet alors de positionner l'électrode mobile à un point d'équilibre. La caractéristique tension-déplacement peut alors être un étalon de transfert très stable si le dispositif est fabriqué à partir d'un monocristal de silicium qui présente une grande stabilité mécanique.

\section{Principe des références de tension MEMS}

Dans le cas des références de tension électrique, on utilise le phénomène du «pull-in » dans un microsystème à capacité variable; phénomène qui, dans une première approximation, limite le déplacement stable de l'électrode mobile à un tiers de la valeur de la distance entre les électrodes $d$. Le pull-in correspond à la tension maximale au-delà de laquelle les deux électrodes vont inévitablement au contact l'une de l'autre (la force électrique dépasse la force mécanique de rappel).

La figure 1 montre un MEMS capacitif à actionnement électrostatique. Quand une tension continue $V_{\text {ext }}$ est 


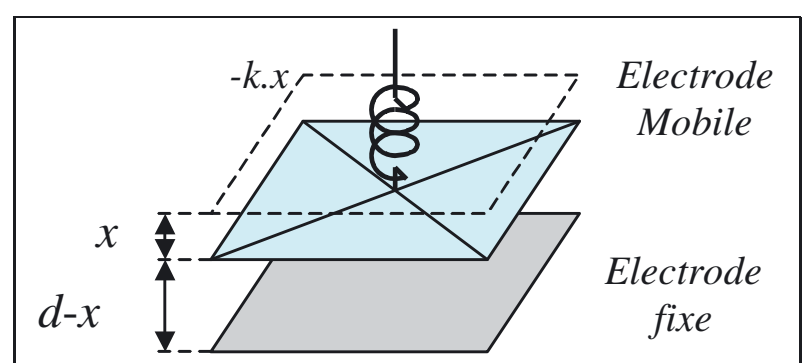

Fig. 1. - Illustration d'un MEMS capacitif à actionnement électrostatique.

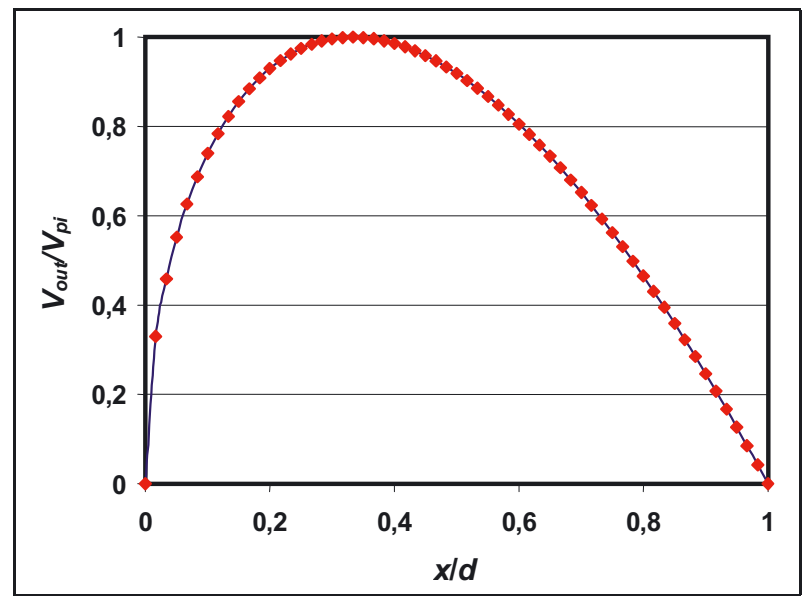

Fig. 2. - Tension aux bornes du MEMS, $V_{\text {out }}$, normalisée à $V_{\mathrm{pi}}$ en fonction du déplacement $x$.

appliquée aux bornes des deux électrodes, une force électrostatique attractive $F_{e}$ apparaît :

$$
F_{e}=\frac{1}{2} V_{\mathrm{ext}}^{2} \frac{\varepsilon A}{(d-x)^{2}},
$$

où $x$ est le déplacement, $d$ la distance inter-électrode à $x=0, C_{0}$ est la capacité à $x=0\left(C_{0}=\varepsilon A / d\right)$, $\varepsilon$ est la constante diélectrique et $A$ la surface de l'électrode.

Cette force électrique tend à rapprocher les électrodes alors que la force mécanique de rappel $F_{m}\left(F_{m}=-k x\right)$ tend à restaurer la position de l'électrode mobile.

La figure 2 montre la tension $V_{\text {out }}$ (normalisée par rapport à $V_{\text {pi }}$ ) aux bornes de la capacité en fonction du déplacement qui présente un maximum en $x=d / 3$, appelé tension de pull-in, $V_{\text {pi }}$ (Eq. 2) :

$$
V_{\mathrm{pi}}=\sqrt{8 k d^{2} / 27 C_{0}}
$$

Dans ce cas, on peut donc utiliser cette tension de pull-in comme référence de tension DC puisqu'une variation relative de la position de l'électrode autour de $x=d / 3$ n'affecte la valeur de $V_{\mathrm{pi}}$ qu'au second ordre (Eq. 3) :

$$
\Delta V_{\mathrm{Pi}} / V_{\mathrm{pi}} \sim-27 / 4(\Delta x / d)^{2}
$$

Le comportement de la tension aux bornes de la capacité décrite par la figure 2 est cependant complètement

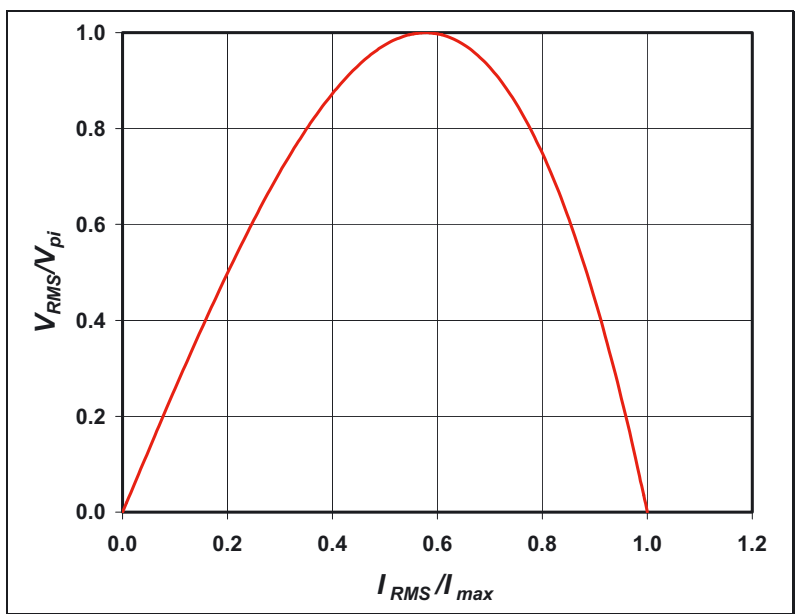

Fig. 3. - Tension RMS aux bornes du MEMS, normalisée à $V_{\mathrm{pi}}$, en fonction du courant RMS normalisé par rapport au courant maximum.

théorique puisque dans le cas de l'actionnement par une tension continue, le système devient instable au-delà de la position $x=d / 3$. Plusieurs techniques permettent alors de stabiliser l'électrode mobile au-delà de la position de pull-in : boucle de contre-réaction de tension DC [14], capacités placées en séries avec le composant [15-17], contrôle de la charge avec des capacités commutables $[18,19]$ ou bien à travers un courant [20].

Si maintenant l'actionnement de la structure illustrée en figure 1 est assuré par un courant AC sinusoïdal d'amplitude $I_{\text {RMS }}$ et de pulsation $\omega$, la force électrique devient alors indépendante de la position $x$ de l'électrode mobile, et l'amplitude de la tension AC aux bornes des électrodes peut s'écrire selon (4) [3] :

$$
V_{\mathrm{AC}}=\frac{I_{\mathrm{RMS}}}{\omega C_{0}}\left[1-\frac{4}{27}\left(\frac{I_{\mathrm{RMS}}}{\omega C_{0} V_{\mathrm{pi}}}\right)^{2}-\frac{F_{m}}{k d}\right],
$$

où $F_{m}$ représente l'ensemble des forces mécaniques extérieures.

L'amplitude de cette tension présente, en fonction du courant $I_{\text {RMS }}$, un maximum (Fig. 3) :

$$
V_{\mathrm{AC}}^{\max }=V_{\mathrm{pi}}=\sqrt{8 k d^{2} / 27 C_{0}} .
$$

Ce maximum peut être utilisé comme une référence de tension AC stable, puisque sa variation relative en ce point dépend seulement du carré de celle du courant (Eq. 5) :

$$
\Delta V_{\mathrm{RMS}} / V_{\mathrm{pi}} \sim-3 / 2\left(\Delta I / I_{\max }\right)^{2}
$$

Dans ce cas, pour minimiser l'influence des forces mécaniques, ces références devront être mises en œuvre à des fréquences très supérieures à la fréquence de résonance du système $f_{0}$ :

$$
f_{0}=\frac{\sqrt{k / m}}{2 \pi} .
$$


Suivant cette approche du pull-in, les travaux réalisés dans le cadre du projet européen EMMA (ElectroMechanical Microcomponents for Precision Applications) portent sur l'étude et le développement de composants MEMS très stables et la mise au point de leur processus de fabrication [21]. Ainsi, la stabilité de ces structures MEMS a été étudiée théoriquement et expérimentalement. Un des résultats marquants est lié à la mise au point d'une référence de tension $\mathrm{AC}$ de $9 \mathrm{~V}$, sur la base d'un MEMS accéléromètre commercial, montrant une stabilité de la tension à $100 \mathrm{kHz}$ de l'ordre de $2 \times 10^{-6}$ sur trois semaines de mesure [22]. Cette stabilité a toutefois été obtenue en appliquant des corrections dues aux effets de température, d'humidité et de pression, mais surtout par la compensation de la tension continue dite «built-in voltage » générée à aux interfaces métal-semi-conducteur dans le système.

Afin d'obtenir les performances de stabilité requises pour les domaines de la métrologie et de l'instrumentation de précision (quelques $10^{-6}$ par an), il est important de concevoir des architectures ayant une faible sensibilité à l'égard des contraintes mécaniques et à la dispersion géométrique, et de développer des procédés technologiques assurant des contraintes résiduelles faibles et une répétabilité élevée. Nous présentons ici les premiers résultats de développement de MEMS pour réaliser des références de tension $\mathrm{AC}$ : des premiers modèles de test pour valider le procédé technologique et pour développer l'électronique de commande, et un deuxième modèle aux caractéristiques améliorées.

\section{Conception et fabrication des MEMS}

La stabilité de la tension de référence $\mathrm{AC}$ (tension de pull-in) à base de MEMS dépend en principe de la stabilité de la constante de raideur $k$ et des propriétés géométriques du dispositif (notamment l'écartement $d$ ). La constante $k$ peut être très stable si le matériau utilisé est un monocristal de silicium. Cependant, une régulation de la température du MEMS est alors indispensable compte tenu de la dépendance en température du module de Young et donc de la constante de raideur. Les contraintes mécaniques natives dans le matériau ou induites par le procédé de fabrication ou le montage du composant MEMS constituent également une limitation à cette stabilité. Par ailleurs, les effets de charge des surfaces de silicium sont à l'origine de variations de la tension du MEMS, causant des instabilités qui peuvent être éliminées par la métallisation des électrodes par dépôt d'aluminium ou d'or. D'autres causes d'instabilité proviennent alors des tensions continues induites aux interfaces métal/semi/conducteur (built-in voltage), dépendant de la température, et qui peuvent être compensées par différentes techniques.

Il apparaît donc que le choix du procédé de fabrication (micro-usinage de surface ou de volume) et des matériaux (membranes silicium ou métalliques) qui conditionne les caractéristiques mécaniques finales des microsystèmes sera crucial. Nous avons donc conçu

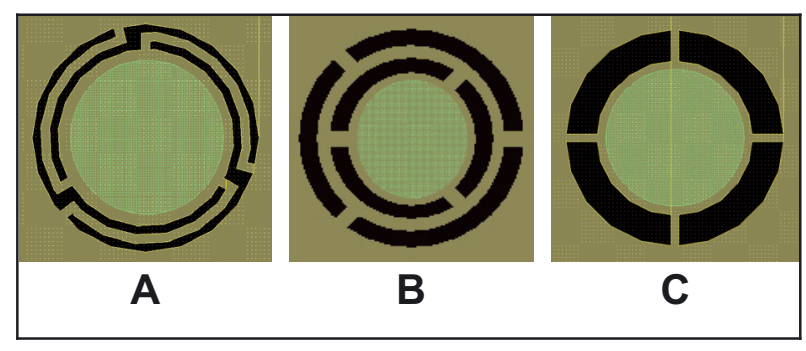

Fig. 4. - Dessins des trois structures MEMS de tests.

plusieurs modèles de microsystèmes, en commençant par des structures de test à membrane circulaire en silicium, puis des modèles améliorés pour évaluer leurs performances par rapport aux différents paramètres à l'origine des instabilités des MEMS.

\subsection{Les structures MEMS modélisées}

La définition de la géométrie des microsystèmes à réaliser doit tenir compte des contraintes suivantes :

- la valeur de la capacité variable doit être supérieure aux capacités parasites du système et avoir une plage de variation compatible avec la détection du pull-in;

- la tension de pull-in doit avoir la valeur de la tension de référence visée;

- la fréquence de résonance doit être suffisamment basse $(<50 \mathrm{kHz})$; ce qui implique des dimensions importantes du système.

\subsubsection{Modèle $n^{\circ} 1$}

Les premières structures MEMS de test ont été réalisées avec l'objectif de tester les performances d'un procédé SOI industriel et des architectures MEMS spécifiques qui peuvent répondre aux problèmes liés à la réalisation technologique elle-même et aux instabilités mécaniques et électriques dans les structures MEMS pour les références de tension. Nous avons donc modélisé trois structures MEMS basées sur un disque circulaire en silicium (membrane mobile) suspendu à des ressorts ayant différentes configurations afin d'assurer le meilleur contrôle du déplacement vertical de l'électrode mobile dans le mode piston. Ces structures ont été modélisées avec le logiciel de simulation Coventor et l'outil de conception spécifique fourni par la société Tronics Microsystems.

La figure 4 représente les schémas des structures MEMS. Le premier échantillon (A) est un disque circulaire de rayon $500 \mu \mathrm{m}$ suspendu par trois ressorts placés à $120^{\circ}$, de longueur $1300 \mu \mathrm{m}$ et de largeur $10 \mu \mathrm{m}$. La forme courbée de ces ressorts permet un gain important sur la surface totale occupée par le MEMS. L'échantillon (B) est très similaire au précédent avec les mêmes dimensions de la membrane silicium et des ressorts. Dans ce dernier cas, les trois ressorts sont attachés ensemble 


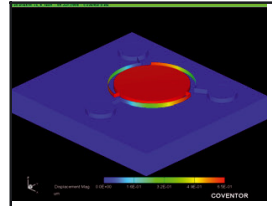

A

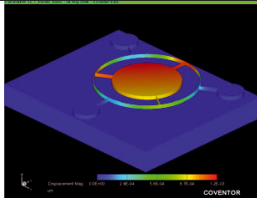

B

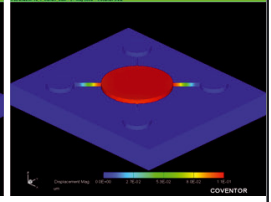

C
Fig. 5. - Les trois structures MEMS de tests modélisées sous Coventor.

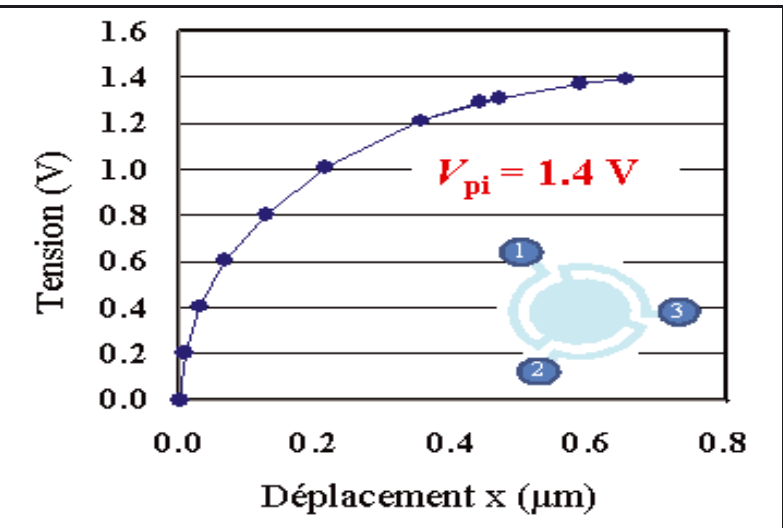

Fig. 6. - Le déplacement $x$ est calculé pour chaque tension continue de polarisation appliquée au MEMS A.

formant un anneau circulaire de largeur $10 \mu \mathrm{m}$, ceci augmente légèrement la rigidité du système et donc la tension de pull-in. L'avantage de cette structure est un meilleur contrôle de la guidance verticale de la membrane mobile. Le dernier dispositif $(\mathrm{C})$ utilise la même membrane silicium de rayon $500 \mu \mathrm{m}$ suspendue cette fois-ci par quatre ressorts droits de longueur $360 \mu \mathrm{m}$ et de largeur $10 \mu \mathrm{m}$. Toutes ces structures ont été conçues pour avoir une capacité nominale $C_{0}$ de l'ordre de quelques picofarads et des fréquences de résonance propres inférieures à $100 \mathrm{kHz}$.

La figure 5 représente les trois structures MEMS modélisées sous CoventorWare. Pour calculer la tension de pull-in de chaque structure, on calcule le déplacement de la membrane mobile sous l'effet de la force électrostatique générée par la tension continue de polarisation appliquée entre les deux électrodes formées par la membrane mobile et le substrat. Les simulations ont été réalisées en tenant compte des dimensions du substrat SOI et notamment d'une distance $d=2 \mu \mathrm{m}$. Dans ce cas, la tension de pull-in correspond à la tension maximum quand le déplacement de la membrane est de 0,66 $\mu \mathrm{m}$ $(d / 3)$. La figure 6 traduit la variation de la position $x$ en fonction de la tension continue de polarisation pour la structure MEMS A, ce qui permet une estimation de la tension de pull-in pour cette structure.

À partir des résultats obtenus de la tension de pullin des différentes structures, on peut alors calculer les constantes de raideur $k$ des différents ressorts et les fréquences de résonance. Le tableau 1 résume ces caractéristiques pour les trois types de MEMS modélisés. Les capacités $C_{0}$ ne tiennent pas compte ici des capacités

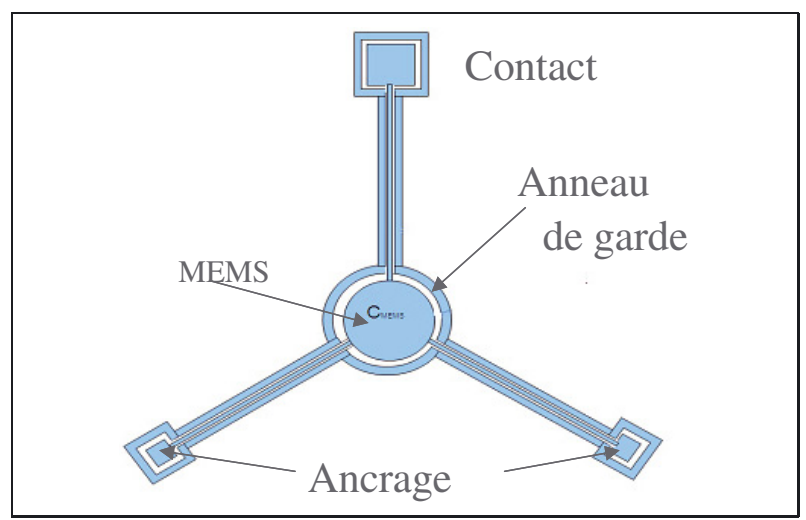

Fig. 7. - MEMS selon le modèle ${ }^{\circ} 2$.

Tableau 1

Caractéristiques des trois types de MEMS modélisés sous CoventorWare.

\begin{tabular}{|c|c|c|c|c|}
\hline Structure & $\boldsymbol{C}_{\mathbf{0}}(\mathbf{p F})$ & $\boldsymbol{k}\left(\mathbf{N} \cdot \mathbf{m}^{-1}\right)$ & $\boldsymbol{f}_{\mathbf{0}}(\mathbf{k H z})$ & $\boldsymbol{V}_{\mathbf{p i}}(\mathbf{V})$ \\
\hline $\mathbf{A}$ & 5,0 & 122 & 5,3 & 1,4 \\
\hline $\mathbf{B}$ & 3,5 & 2338 & 23,1 & 28 \\
\hline $\mathbf{C}$ & 3,4 & 23564 & 74 & 90 \\
\hline
\end{tabular}

Tableau 2

Dimensions des MEMS du modèle $\mathrm{n}^{\circ} 2$.

\begin{tabular}{|c|c|c|c|}
\hline $\begin{array}{c}\text { Type de } \\
\text { structures }\end{array}$ & $\begin{array}{c}\text { Diamètre } \\
(\boldsymbol{\mu m})\end{array}$ & $\begin{array}{c}\text { Longueur } \\
(\boldsymbol{\mu m})\end{array}$ & $\begin{array}{c}\text { Largeur } \\
\text { Largeur }(\boldsymbol{\mu m})\end{array}$ \\
\hline MEMS 5 V & 2210 & 1072 & 10 \\
\hline MEMS 10 V & 2210 & 884 & 10 \\
\hline
\end{tabular}

oxyde dues aux points d'ancrage des membrane mobiles qui sont des capacités parasites.

\subsubsection{Modèle $n^{\circ} 2$}

Suite aux premiers modèles de MEMS qui ont servi de structures de test, une deuxième structure a été réalisée sur la base d'une membrane sous forme de disque cylindrique porté par trois ressorts droits (Fig. 7). Nous avons ainsi augmenté la valeur de la capacité nominale de la structure et réduit celles des capacités parasites et notamment celles liées aux points d'ancrage de l'électrode mobile et des contacts électriques. Pour cela, le diamètre du disque a été doublé pour atteindre $2210 \mu \mathrm{m}$ correspondant à une capacité de $23 \mathrm{pF}$, contre $5 \mathrm{pF}$ pour les structures de test, et seul un point de contact électrique est prévu pour l'électrode mobile. Par ailleurs, un anneau de garde de largeur $65 \mu \mathrm{m}$ entoure toute la structure pour garder électriquement l'électrode mobile du reste du silicium du substrat SOI.

Deux structures MEMS ont été réalisées en ciblant deux tensions de pull-in de $5 \mathrm{~V}$ et $10 \mathrm{~V}$. Le tableau 2 donne les dimensions de chacune de ces deux structures.

Ces deux structures ont été modélisées sous CoventorWare. La figure 8 donne la tension de pull-in pour la structure $10 \mathrm{~V}$ et le tableau 3 résume les caractéristiques calculées pour les deux MEMS. 


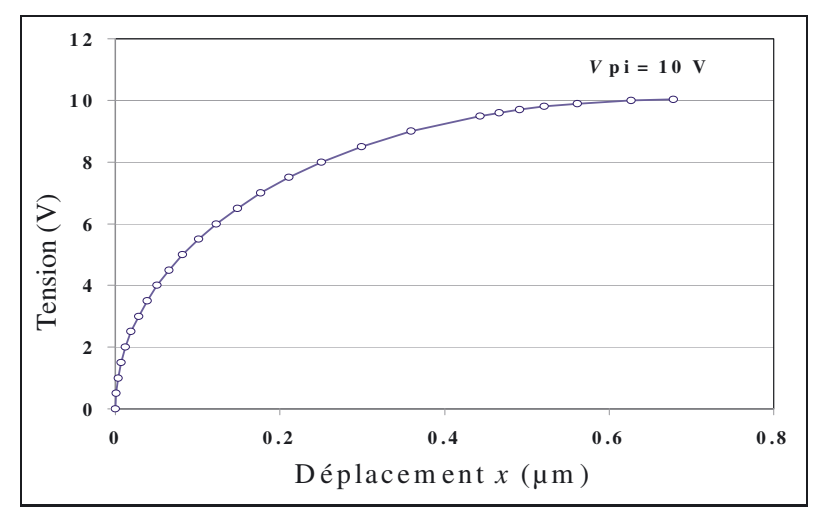

Fig. 8. - Le déplacement $x$ est calculé pour chaque tension continue de polarisation appliquée au MEMS $10 \mathrm{~V}$.

Tableau 3

Caractéristiques des deux MEMS (5 V et $10 \mathrm{~V}$ ) modélisés sous CoventorWare.

\begin{tabular}{|c|c|c|c|c|}
\hline Structure & $\boldsymbol{C}_{\mathbf{0}}(\mathbf{p F})$ & $\boldsymbol{k}\left(\mathbf{N} \cdot \mathbf{m}^{-1}\right)$ & $\boldsymbol{f}_{\mathbf{0}}(\mathbf{k H z})$ & $\boldsymbol{V}_{\mathbf{p i}}(\mathbf{V})$ \\
\hline MEMS 5 V & 22,8 & 437 & 10 & 5,5 \\
\hline MEMS 10 V & 22,8 & 1437 & 18 & 10 \\
\hline
\end{tabular}

\subsection{Fabrication des microsystèmes}

\subsubsection{Procédé Tronics microsystems}

Pour la réalisation technologique, nous avons choisi un procédé industriel disponible chez la société Tronics microsystems sous une offre Multi Project Wafer offer (MPW). Ce procédé technologique qui est bien maîtrisé permet de minimiser les contraintes et les gradients de contrainte, d'avoir un meilleur contrôle des dimensions des structures et d'assurer la répétabilité et la robustesse de la fabrication. Il consiste en un micro-usinage de surface à partir d'un substrat SOI qui est constitué par :

- une couche monocristalline de silicium mécaniquement active (top layer), d'épaisseur de $60 \mu \mathrm{m}$ et ayant d'excellentes caractéristiques mécaniques (elle peut tolérer jusqu'à $10^{10}$ cycles sans aucune fissure ou fatigue [23]):

- une couche d'oxyde de silicium $\left(\mathrm{SiO}_{2}\right)$ dont l'épaisseur de $2 \mu \mathrm{m}$ définit la distance entre les deux électrodes de la capacité variable des structures MEMS à déplacement vertical ;

- un substrat de silicium monocristallin d'épaisseur $450 \mu \mathrm{m}$ et qui sert également d'électrode fixe dans ce dernier cas. Pour ce substrat SOI, la résistivité du silicium est de $0,02 \Omega \cdot \mathrm{cm}$ obtenue grâce à un fort dopage type-p de bore de $2 \times 10^{18} \mathrm{~cm}^{-3}$.

La figure 9 illustre le déroulement du procédé utilisé pour la réalisation des structures. D'abord la couche active est gravée pour réaliser la membrane mobile circulaire suspendue par des ressorts. Ensuite, les passages des contacts sont réalisés avec une métallisation or. Après une gravure sélective de la couche d'oxyde, la membrane silicium ainsi que les ressorts sont libérés du substrat.

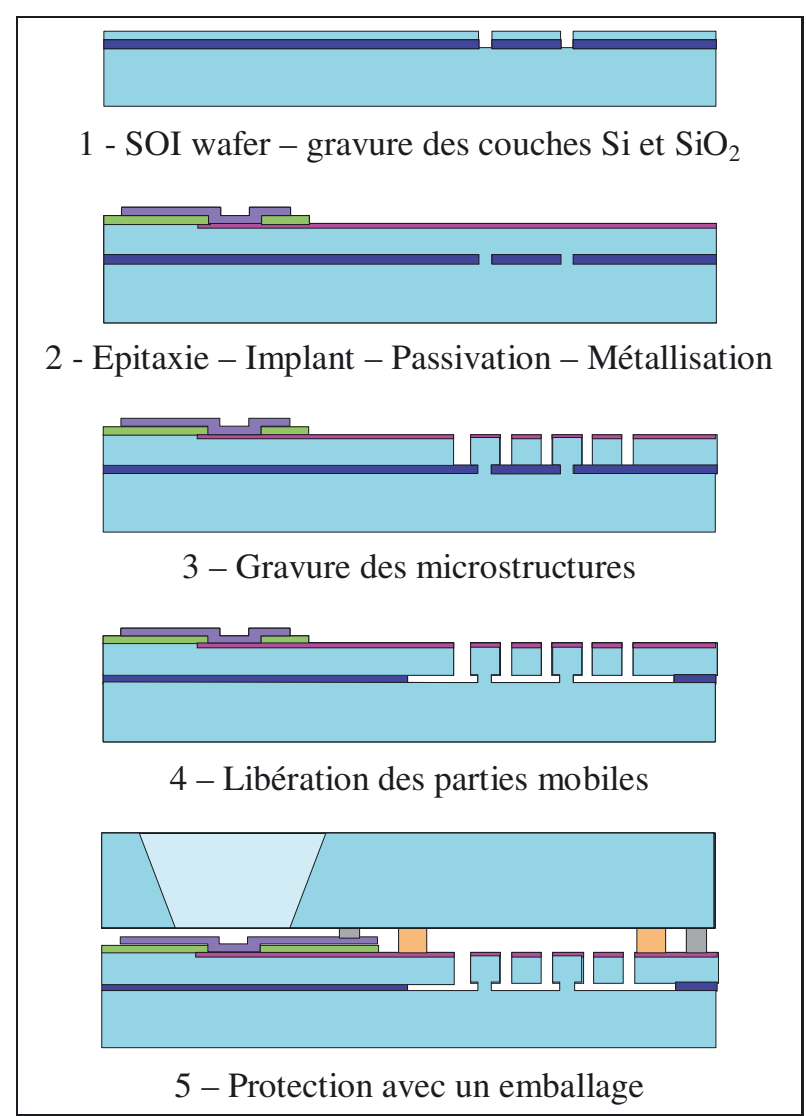

Fig. 9. - Procédé MPW de Tronics utilisé pour réaliser les dispositifs MEMS.

Pour protéger les MEMS ainsi réalisés, les puces sont recouvertes d'un emballage scellé au niveau du substrat en silicium. La structure finale est obtenue par l'assemblage du substrat SOI contenant le MEMS et un substrat en silicium agissant comme un capot pour protéger le composant MEMS (Fig. 10). Cet emballage peut être hermétique sous vide ou non hermétique. Les contacts électriques sont réalisés après l'assemblage des deux substrats via des trous métallisés.

\subsubsection{MEMS réalisés}

Les trois premières structures MEMS ont été réalisées en utilisant le procédé Tronics. Ces structures sont présentées sur la figure 11 sous la forme de photographies réalisées sous microscope électronique à balayage (MEB). Sur la figure 12 on peut voir quelques détails de cette réalisation comme les points d'ancrage des structures, les contacts électriques, les ressorts et les trous dans les membranes mobiles servant pour l'étape de gravure.

Pour contrôler la qualité du micro-usinage et des gravures, des motifs de test ont été réalisés sous forme de poutre et d'écartement de largeur respectivement $3 \mu \mathrm{m}$ et $4 \mu \mathrm{m}$. La mesure de ces paramètres sur une dizaine de motifs correspondant à une dizaine de puces, montre une dispersion inférieure à $0,2 \mu \mathrm{m}$, ce qui indique une très bonne maîtrise du procédé technologique et une très bonne répétabilité. 


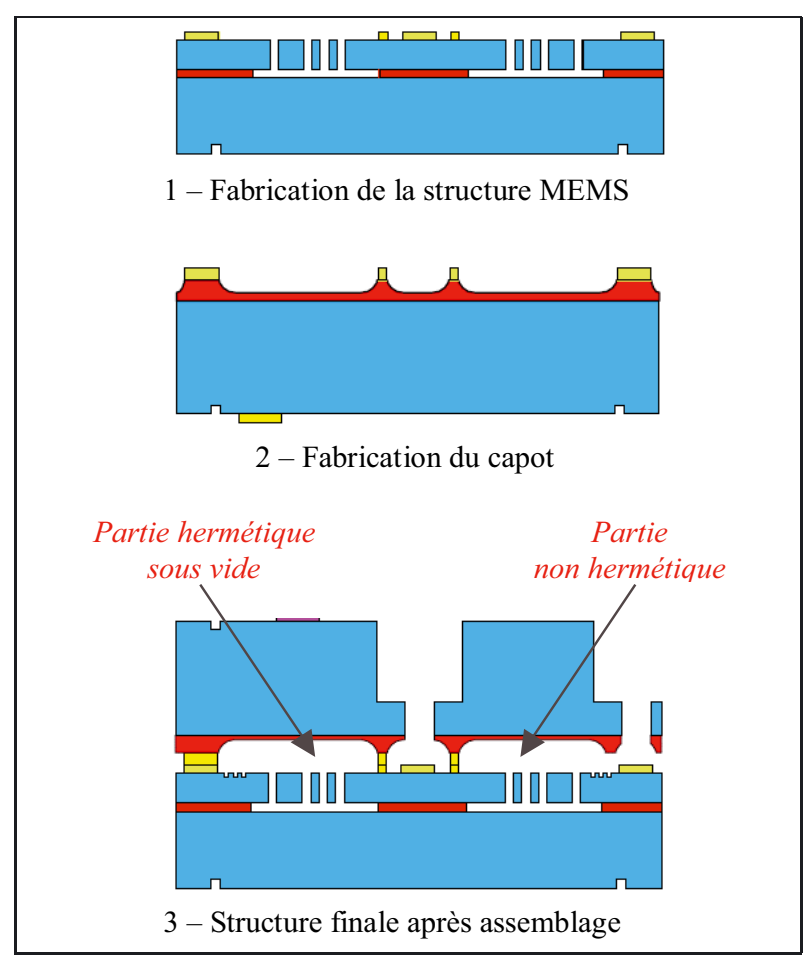

Fig. 10. - Emballage des dispositifs MEMS.

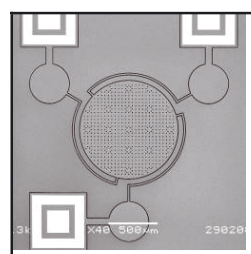

A

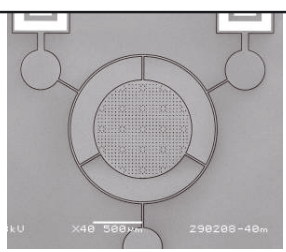

B

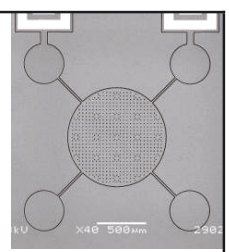

C
Fig. 11. - Photographies MEB des trois structures MEMS de test.

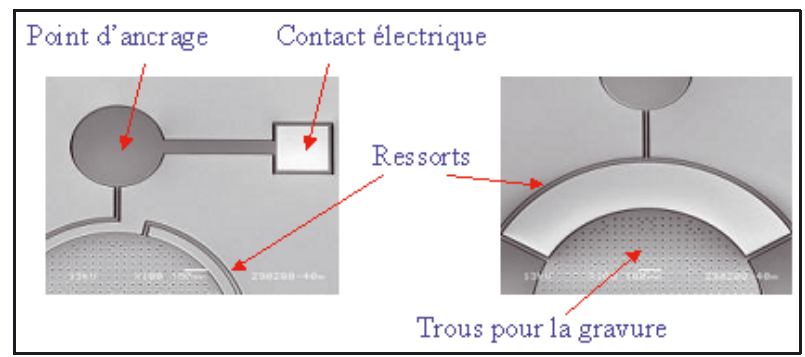

Fig. 12. - Détails des structures MEMS de test.

La figure 13 montre la puce finale de surface $(7 \times$ 8) $\mathrm{mm}^{2}$ contenant six composants MEMS, trois avec un emballage hermétique et trois autres avec un emballage non hermétique, collée sur un support TO8 avec une colle époxy conductrice.

Avec ce même procédé MPW de Tronics, nous avons ensuite réalisé les structures du modèle $\mathrm{n}^{\circ} 2$ avec des membranes circulaires en silicium munies d'un anneau de garde qui peut être mis à un potentiel choisi. La figure 14 montre une photographie réalisée au MEB d'une

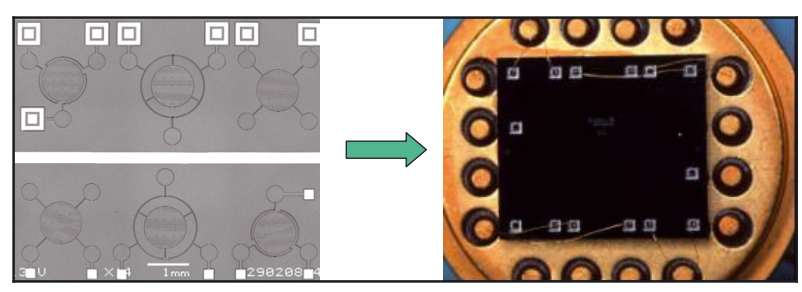

Fig. 13. - Puce contenant les composants MEMS et collée sur un support TO8.

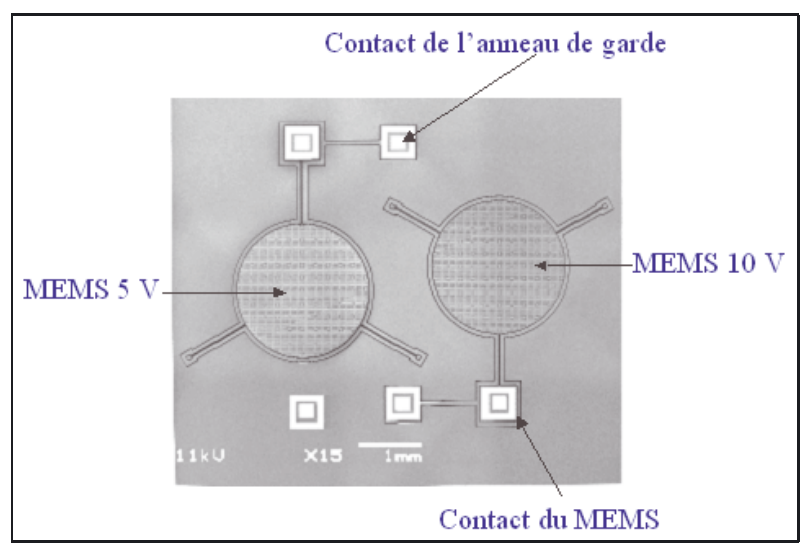

Fig. 14. - Puce contenant les composants MEMS du modèle $n^{\circ} 2$.

puce contenant les deux dispositifs MEMS de $5 \mathrm{~V}$ et $10 \mathrm{~V}$. Des puces hermétiques et non hermétiques ont été fabriquées.

\section{Electronique de commande}

Bien que le principe d'actionnement et de contrôle d'un MEMS capacitif dans le cas de la référence de tension $\mathrm{AC}$ soit relativement simple, l'électronique à développer doit être soigneusement élaborée. Ceci pour ne pas induire du bruit, compte-tenu des valeurs des capacités de travail, comparables aux capacités de fuite du système lui-même (quelques picofarads). Cette électronique doit stabiliser la position du système, grâce à des dispositifs de contre-réaction, suite à des déplacements dus à des forces électriques ou mécaniques (phénomène de pull-in ou d'amortissement). Les composants électroniques utilisés doivent être préalablement caractérisés et sélectionnés, notamment les amplificateurs opérationnels qui seront montés en intégrateurs avec la capacité MEMS et devront avoir un gain très stable. Deux solutions de montage ont été étudiées : un circuit stabilisé par la capacité MEMS mais dont l'ajustement du gain est manuel, et un circuit plus sophistiqué capable d'ajuster automatiquement la tension de sortie du MEMS à sa valeur maximale.

La tension aux bornes de la capacité MEMS au point de pull-in est maximale et dépend peu du courant et de la fréquence. Pour des petites variations, seul un terme de second ordre va être perceptible : une erreur sur le rapport $I / \omega$ de l'ordre de $10^{-4}$ n'entraînera sur la tension qu'une erreur de l'ordre de $10^{-8}$. Donc, si les constantes caractéristiques du dispositif, $C_{0}$ et $V_{\mathrm{pi}}$ sont stables, la 


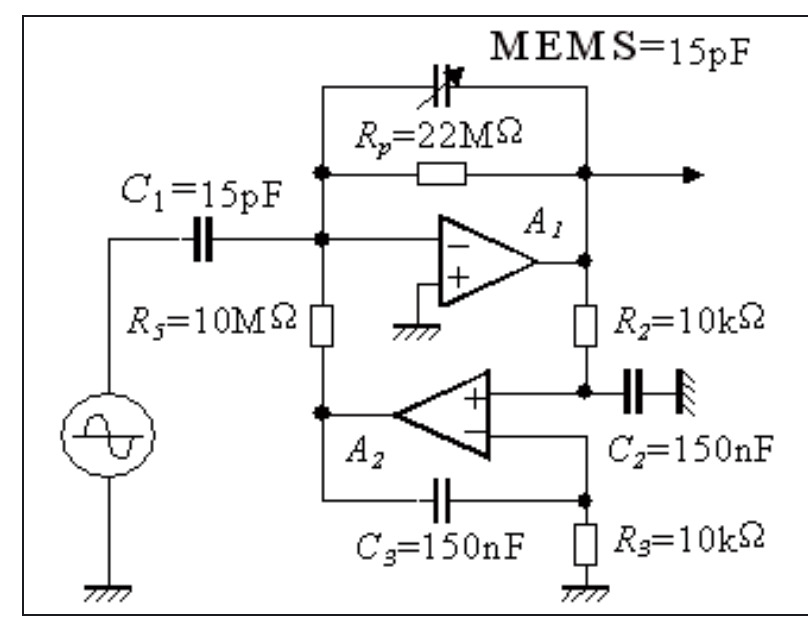

Fig. 15. - Montage avec contre-réaction sur la tension continue de sortie.

tension aux bornes du condensateur est stable et peut servir de référence de tension $\mathrm{AC}$ pour la métrologie. Notons tout de même qu'il est aisé d'avoir une excellente stabilité de la fréquence, à l'aide d'un oscillateur à quartz, mais qu'il n'est pas facile de maîtriser avec précision un courant alternatif à $100 \mathrm{kHz}$ ou $1 \mathrm{MHz}$, et de maintenir fixe le rapport entre le courant et la fréquence pour rester au point de pull-in. Il est donc important de disposer d'un asservissement automatique de cette tension de référence.

Le premier montage électronique développé est schématisé sur la figure 15 où le dispositif MEMS est placé en contre-réaction de l'amplificateur principal $A_{1}$ qui doit avoir un grand produit gain-bande. Cependant, les amplificateurs offrant un grand produit gain-bande ont rarement des offsets (tensions électriques de décalage) et des courants de polarisation faibles. L'usage d'un amplificateur «de précision », c'est à dire à très faible tension de décalage et très faible courant de polarisation est alors nécessaire pour imposer une tension continue nulle en sortie du MEMS. Sur la figure 15, la tension continue de sortie est maintenue à zéro par l'amplificateur auxiliaire de précision $A_{2}$ en dépit d'une très grande résistance de contre-réaction $\left(R_{p}=22 \mathrm{M} \Omega\right)$. Le courant de polarisation circule dans $R_{5}$, et la tension continue en sortie de $A_{2}$ peut être de plusieurs volts. La tension continue d'entrée du MEMS est égale à la tension de décalage de l'amplificateur principal $A_{1}$. La courbe de réponse «passebande » est celle du signal, où la coupure haute (environ $2 \mathrm{MHz}$ ) dépend des paramètres de l'amplificateur $A_{1}$ et de sa charge. La courbe «passe-bas » est la réponse à la tension de décalage ou de bruit de l'amplificateur $A_{2}$.

Pour contrôler automatiquement la position du MEMS au point de pull-in, une solution envisagée, testée dans le cadre du projet européen EMMA, est d'asservir le point de fonctionnement au moyen d'une modulation d'amplitude (AM) du signal radiofréquence par un petit signal sinusoïdal à très basse fréquence. En effet, un tel asservissement élimine de nombreuses sources d'erreur et seule subsiste l'erreur de gain (qualité de l'amplificateur). Dans cette approche, un modulateur effectue le

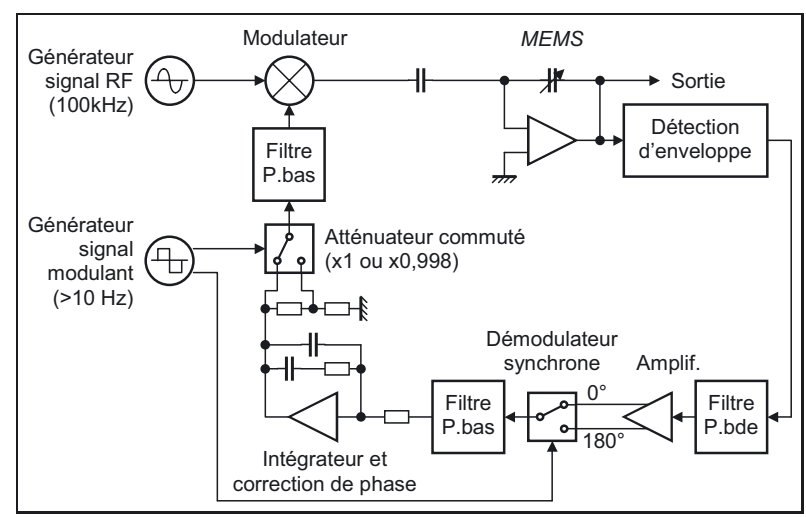

Fig. 16. - Modulation d'amplitude avec un signal carré.

produit du signal RF entrant par la somme d'une tension continue et d'une petite tension alternative $(1,3 \mathrm{~Hz}$ dans le cas du projet EMMA). Ce signal a donc une amplitude moyenne qui dépend de cette tension continue, et subit une petite modulation d'amplitude de faible profondeur. Si l'amplitude moyenne est correcte, il n'y a pas de composante très basse fréquence $(1,3 \mathrm{~Hz})$ dans l'amplitude détectée en sortie. S'il y a une erreur positive ou négative, il apparaît une tension alternative à $1,3 \mathrm{~Hz}$ en phase ou en opposition de phase avec la modulante : la courbe $U=f(I)$ change de pente. Une détection synchrone et un filtre passe-bas permettent d'obtenir une tension d'erreur qui après intégration constitue la tension continue de commande.

Dans notre cas, nous avons étudié la possibilité de réaliser la modulation d'amplitude par un signal carré. Avec une telle modulation, dite ASK (Amplitude Shift Keyed), le courant ne prend que deux valeurs fixes $I_{\mathrm{pi}} \pm$ $\Delta_{I}$, le point de fonctionnement ne décrit donc plus l'arc de la courbe $U=f(I)$ et il n'y a plus de modulation résiduelle au double de la fréquence (sauf un résiduel d'impulsions). Cette nouvelle approche présente deux avantages :

- il n'y a aucune gêne pour des systèmes échantillonnés ou impulsionnels ;

- les circuits de filtrage deviennent très simples donc apportent moins de déphasage dans la boucle d'asservissement. Pour une même fréquence modulante, la bande passante de boucle peut être plus grande.

Par ailleurs, plutôt que d'additionner la modulante et la tension de commande intégrée, il faut la multiplier par l'enveloppe de modulation choisie, puis moduler le signal avec ce produit. Avec une modulante sinusoïdale, cela suppose un multiplieur supplémentaire. Avec un signal carré, le produit est effectué avec un simple commutateur analogique CMOS, suivi d'un filtre passe-bas pour contrôler le contenu harmonique du signal modulant. Le démodulateur synchrone est également un commutateur analogique, beaucoup plus stable en courant continu qu'un multiplieur analogique. Le module de référence de tension à régulation par modulation d'amplitude est présenté par le synoptique de la figure 16. 


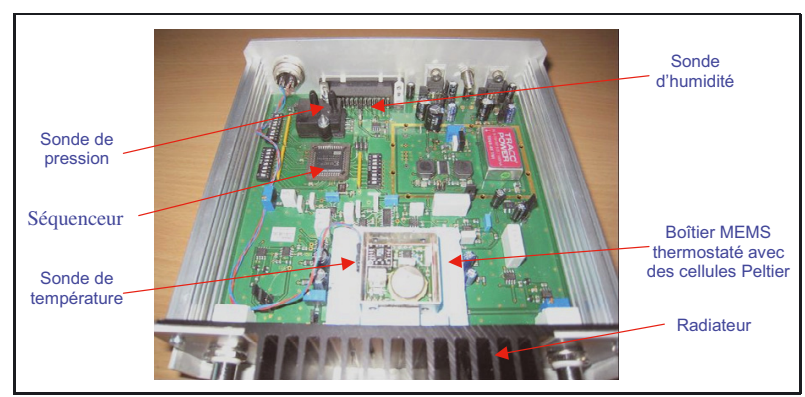

Fig. 17. - Photographie du module électronique complet AM de $3^{\mathrm{e}}$ génération.

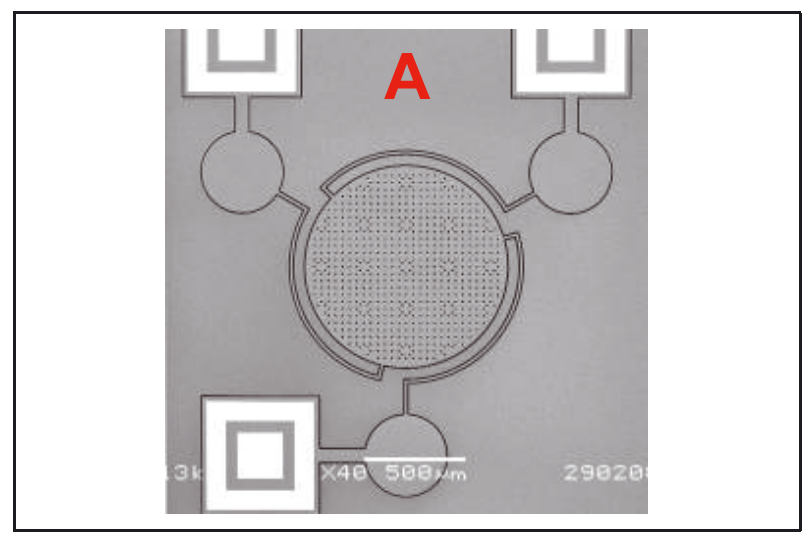

Fig. 18. - Photo du MEMS A issu du modèle ${ }^{\circ} 1$.

Plusieurs montages électroniques ont été développés avec des améliorations successives. La figure 17 montre la version $\mathrm{AM}$ de troisième génération qui intègre également une régulation de température du MEMS.

\section{Résultats expérimentaux}

\subsection{MEMS du modèle $n^{\circ} \mathbf{1}$}

Des caractérisations complètes $C$ - $V$ ont été réalisées sur les premiers prototypes MEMS développés dans le cadre de cette étude. Les premières mesures ont montré que seule la structure A de la figure 18 avait une tension de pull-in suffisamment basse pour être compatible avec l'électronique développée, limitée par la tension maximale de sortie que peut délivrer l'amplificateur principal de contre-réaction.

La figure 19 montre la caractéristique $C$ - $V$ de la structure A à la fréquence de $100 \mathrm{kHz}$ pour une tension de polarisation variant de $-2,5 \mathrm{~V}$ à $2,5 \mathrm{~V}$. On peut tout d'abord remarquer la valeur de la capacité au repos (à $0 \mathrm{~V}$ ) est voisine de $22 \mathrm{pF}$. Rappelons que la capacité nominale $C_{0}$ est de l'ordre $5 \mathrm{pF}$. La différence provient essentiellement des capacités parasites des points d'ancrage de la structure sur le substrat silicium et des trois plots de contacts électriques. Ces capacités parasites sont en parallèle avec la capacité MEMS et peuvent être assez gênantes pour notre application. Ces considérations ont été prises en compte pour l'élaboration des MEMS du modèle $n^{\circ} 2$ en

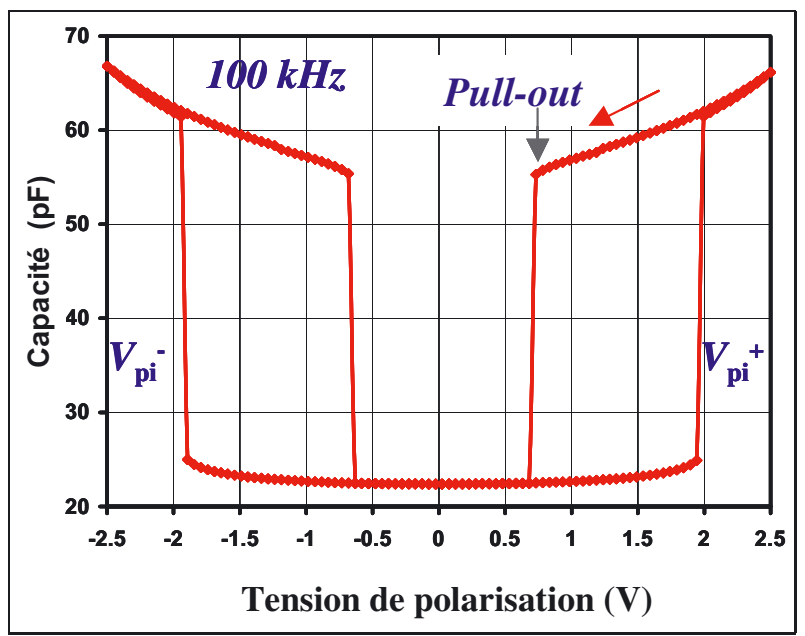

Fig. 19. - Caractéristique $C-V$ du MEMS A à $100 \mathrm{kHz}$.

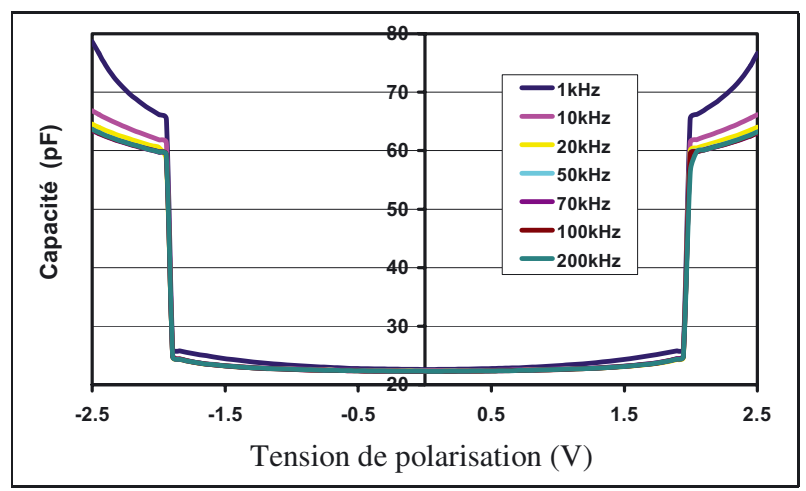

Fig. 20. - Caractéristique $C-V$ du MEMS A à différentes fréquences.

réduisant la taille effective des points d'ancrage et en se limitant à un seul plot de contact électrique pour la membrane mobile.

Par ailleurs, cette caractéristique $C$ - $V$ est totalement symétrique en tension de polarisation positive et négative. En effet, le décalage de la courbe par rapport au zéro n'est que d'une dizaine de millivolts, ce qui traduit des tensions de built-in extrêmement faibles comparées à celles des échantillons décrits dans les publications, où les valeurs peuvent atteindre $1 \mathrm{~V}$. Dans ce dernier cas, les électrodes étaient métallisées, ce qui n'est pas le cas pour les échantillons du LNE. Par ailleurs, on voit clairement un pull-in du MEMS qui se produit à $1,96 \mathrm{~V}$ et une tension de pull-out autour de $0,7 \mathrm{~V}$.

En faisant varier la fréquence du signal $\mathrm{AC}$ de mesure de la capacité de $1 \mathrm{kHz}$ à $200 \mathrm{kHz}$ (Fig. 20), les caractéristiques $C-V$ correspondantes montrent toujours la même tension de pull-in quelle que soit la fréquence.

Les mesures des caractéristiques $U-I$ ont été effectuées avec l'électronique de commande représentée en figure 15 , où le pilotage du MEMS est réalisé de façon manuelle en contrôlant la tension du signal de la source alternative (calibrateur Fluke5720A). Nous avons donc 


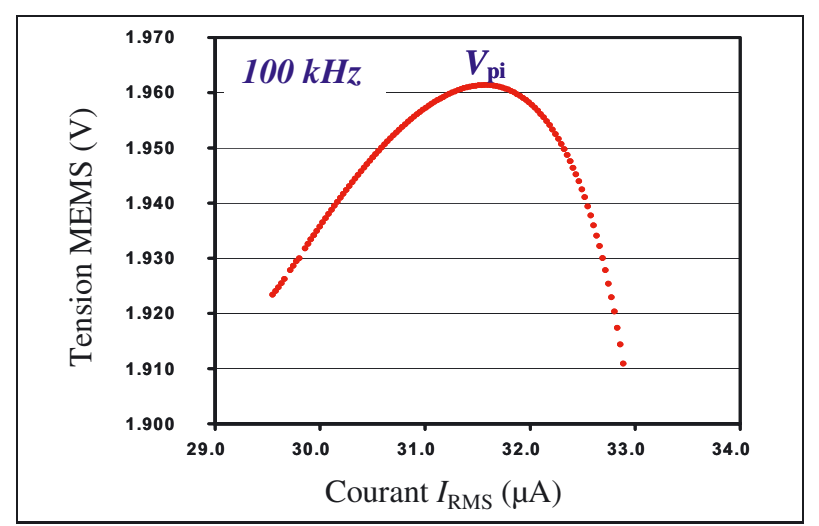

Fig. 21. - Caractéristique $U-I$ du MEMS A à $100 \mathrm{kHz}$.

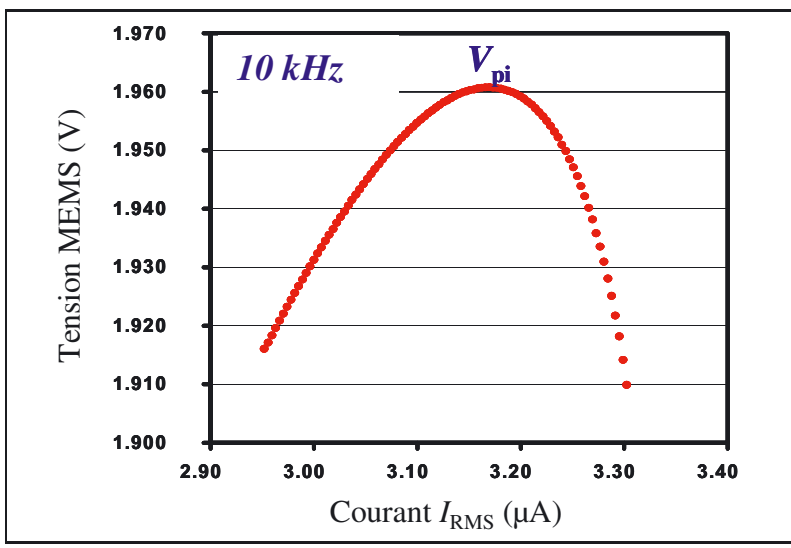

Fig. 22. - Caractéristique $U-I$ du MEMS A à $10 \mathrm{kHz}$

effectué sur le MEMS A des mesures $U-I$ pour des fréquences du courant variant de $10 \mathrm{kHz}$ à $200 \mathrm{kHz}$. La figure 21 montre la variation de la tension $\mathrm{AC}$ aux bornes du MEMS en fonction du courant alternatif d'actionnement à la fréquence de $100 \mathrm{kHz}$. On peut voir que la tension du MEMS passe par un maximum qui se situe exactement à $1,96 \mathrm{~V}$ et qui correspond à la tension de pull-in déterminée sur les courbes $C$ - $V$. Ceci montre clairement que pour un actionnement par un courant alternatif, la membrane mobile du MEMS peut être déplacée au-delà de la position théorique de pull-in. Ce maximum est parfaitement constant pour des fréquences de $10 \mathrm{kHz}$ (Fig. 22) jusqu'à 200 kHz; ce qui montre la possibilité de réaliser des références de tension sur une gamme de fréquences assez étendue.

L'évaluation des caractéristiques des structures MEMS de test du modèle $\mathrm{n}^{\circ} 1$ nous ont permis de collecter un certain nombre d'informations et de dégager des recommandations qui ont servi pour le modèle de la seconde génération :

- nous avons eu beaucoup de défaillances avec les MEMS à ressorts courbes et seuls quelque-uns ont fonctionné. Pour la suite, nous nous sommes donc limités à ne considérer que des structures à ressorts droits. La modélisation et les calculs analytiques sont alors plus pertinents pour prévoir les caractéristiques réellement mesurées ;

- les capacités parasites doivent être fortement réduites en limitant le nombre de plots de contact électrique et en réduisant la taille des points d'ancrage de la structure mobile sur le substrat de silicium. On peut aussi envisager d'augmenter la capacité nominale en tenant compte des limites imposées par les règles du procédé Tronics ;

- pour développer une électronique de commande la plus performante possible, utilisant les meilleurs composants électroniques disponibles sur le marché, notamment les amplificateurs, il faut travailler avec des structures MEMS dont la tension de pull-in est inférieure à $20 \mathrm{~V}$, idéalement entre $1 \mathrm{~V}$ et $10 \mathrm{~V}$. Ceci permet d'évaluer concrètement la stabilité intrinsèque des MEMS sans être gêné par des instabilités de l'électronique de commande elle-même ;

- le procédé SOI Tronics, utilisé pour la fabrication de structures à déplacement vertical, ne permet pas de réaliser une butée mécanique et de métalliser les surfaces internes des électrodes formées par la couche active de silicium et le substrat lui-même. Il faut donc éviter de mettre les deux électrodes en contact (pullin) sous peine d'endommager la structure;

- réduire le nombre de composants MEMS par puce pour éviter d'éventuelles interactions et s'assurer de pouvoir mettre toutes les parties de la puce (silicium non actif, capot) à un potentiel adéquat. Il faut prévoir des plots de contact pour chaque partie.

\subsection{MEMS du modèle $n^{\circ} 2$}

Pour réaliser cette deuxième génération de MEMS, nous avons tenu compte de toutes les remarques issues de l'évaluation des structures MEMS de test. La capacité nominale a ainsi été augmentée pour atteindre $23 \mathrm{pF}$, contre $5 \mathrm{pF}$ précédemment, en doublant le diamètre de la membrane mobile circulaire en silicium. Les capacités parasites ont été également réduites en fixant un seul plot de contact électrique pour l'électrode mobile qui est maintenant munie de points d'ancrage de taille beaucoup plus réduite.

La caractéristique $C-V$ de la structure $5 \mathrm{~V}-\mathrm{HK} 11$ (MEMS $5 \mathrm{~V}$ issu de la puce hermétique K11) à $100 \mathrm{kHz}$ est présentée sur la figure 23. La capacité nominale (à tension nulle) est de $29 \mathrm{pF}$, ce qui signifie que des capacités parasites de l'ordre de $6 \mathrm{pF}$ sont encore présentes. Celles-ci peuvent provenir de la capacité à air entre l'électrode mobile et le capot silicium qui protège le MEMS. En effet, l'écartement entre ces deux parties n'est que de $5 \mu \mathrm{m}$ et la capacité est directement en parallèle avec la capacité MEMS si le substrat et le capot sont au potentiel de la masse. Par ailleurs, le décalage du centre de la courbe correspondant à la tension de built-in n'est que de $0.1 \mathrm{~V}$. Cette valeur est beaucoup plus faible dans les échantillons étudiés que celle mentionnée dans les publications où les électrodes sont métallisées. 


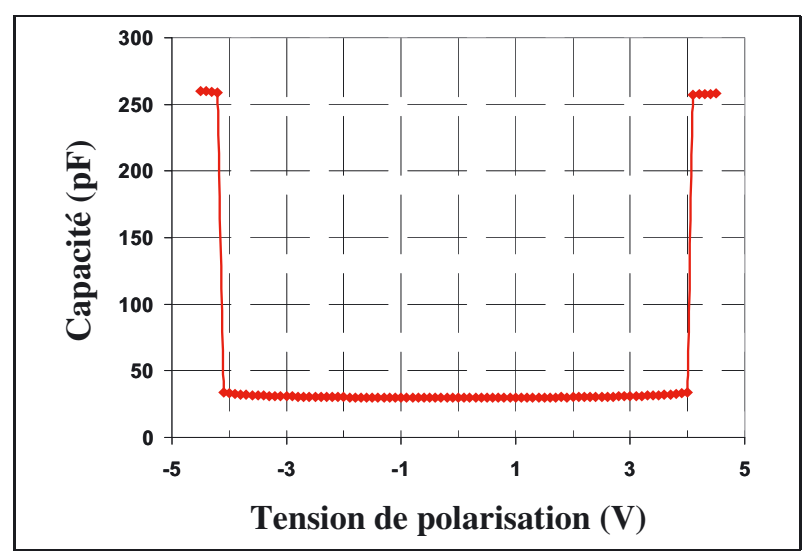

Fig. 23. - Caractéristique $C-V$ du MEMS 5V-HK11 à $100 \mathrm{kHz}$.

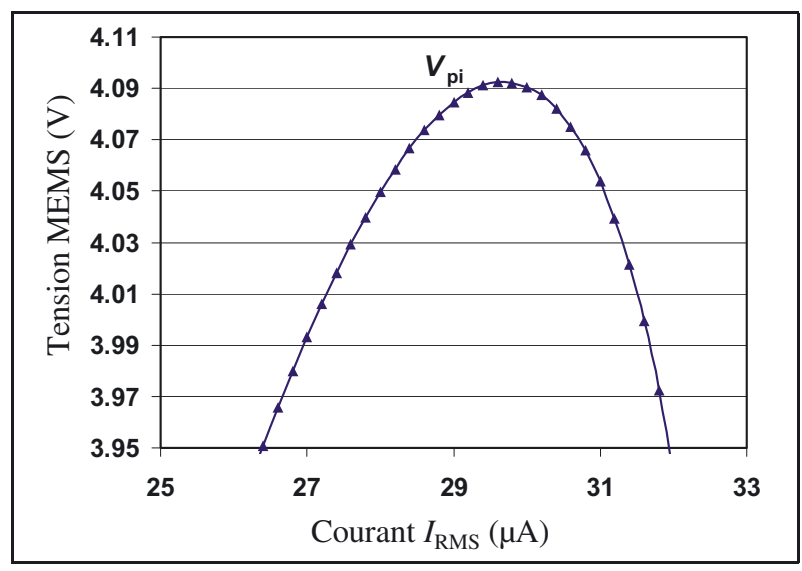

Fig. 24. - Caractéristique $U-I$ du MEMS 5V-HK11 à 100 kHz.

La valeur de la tension de pull-in déterminée à partir de la figure 23 est de 4,095 V. La dispersion de cette valeur sur un certain nombre d'échantillons testés est de quelques dixièmes de volts (tension de pull-in variant de $3,8 \mathrm{~V}$ à 4,6 V). Rappelons que la valeur simulée pour ces structures est de 5,5 V.

La tension de sortie du MEMS 5V-HK11 en fonction du courant d'actionnement à $100 \mathrm{kHz}$ est présentée sur la figure 24. La valeur maximale de la tension est de 4,095 V et correspond exactement à la tension de pull-in déterminée précédemment. C'est cette valeur qui constituera la tension de référence $\mathrm{AC}$ de ce MEMS dont la stabilité doit être parfaitement évaluée et caractérisée en fonction des conditions environnementales.

Pour les MEMS $10 \mathrm{~V}$, la tension de pull-in mesurée est de l'ordre de $8 \mathrm{~V}$. C'est donc le même écart relatif pour les deux types d'échantillons entre les valeurs de pull-in simulées et celles mesurées. Ceci est dû à un maillage non optimisé des structures et à un niveau faible de la précision imposé au calculateur pour réduire le temps de calcul. Ce travail devra être poursuivi pour améliorer notre capacité de simulation de composants complexes.

Nous avons utilisé la technique DLTS (Deep level transient Spectroscopy) pour caractériser le comportement dynamique des MEMS fabriqués. En effet,

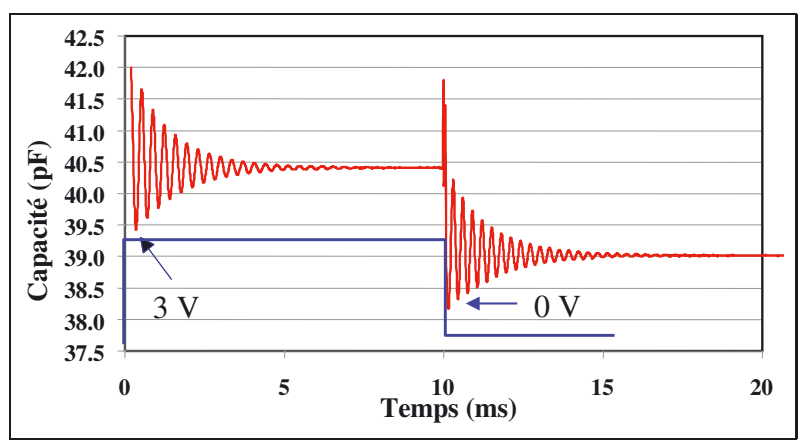

Fig. 25. - Réponse de la capacité du MEMS 5V-HP11 à un échelon de $3 \mathrm{~V}$ en fonction du temps.

cette technique permet d'accéder à des informations sur le comportement des microsystèmes comme les temps de réponse mécanique, l'amortissement ou la fréquence de résonance. Les premières mesures ont été effectuées sur un échantillon du modèle $n^{\circ} 2$ basé sur une membrane circulaire en silicium et à déplacement vertical. La figure 25 représente la réponse d'un MEMS 5V-HP11 (tension de pull-in de $5 \mathrm{~V}$, de la puce référencée HP11) à un échelon de tension continue de $3 \mathrm{~V}$ pendant $10 \mathrm{~ms}$, puis remise à zéro pendant $10 \mathrm{~ms}$.

Des oscillations de la capacité du MEMS, traduisant les oscillations mécaniques de la membrane silicium, sont clairement observées sur une période de l'ordre de $5 \mathrm{~ms}$. Au-delà, la membrane mobile se stabilise à sa position d'équilibre. Le même comportement est observé quand la tension continue est remise à zéro. Ces oscillations indiquent que le système n'est pas complètement amorti, ce qui est en accord avec le fait que la puce HP11 est dans un emballage hermétique sous vide. Notons que cette mesure a été effectuée sous une pression atmosphérique dans la chambre à échantillon dans laquelle il est possible de faire varier la pression jusqu'à réaliser un vide inférieur à $100 \mathrm{~Pa}$ (1 mbar). On peut estimer, à partir des oscillations de la capacité, la fréquence de résonance du système qui est de $2,8 \mathrm{kHz}$. Cette valeur, très basse, permet d'envisager l'utilisation de ces microsystèmes comme références de tension à partir d'une fréquence électrique de $30 \mathrm{kHz}$. Ceci confère à ces microstructures un avantage supplémentaire leur permettant d'être comparées aux convertisseurs thermiques qui présentent les meilleures incertitudes à ces fréquences.

Des mesures de stabilité de la tension de référence AC correspondant au maximum des courbes $U-I$ ont été effectuées sur plusieurs MEMS du modèle $n^{\circ} 2$. Le courant d'actionnement est fourni par un calibrateur (Fluke 5720A) à travers une capacité d'injection de $20 \mathrm{pF}$ et est fixé à la valeur $I_{\max }$. La tension $V_{\mathrm{pi}}$ est alors mesurée par un voltmètre numérique (Agilent 3458A) raccordé et configuré dans le mode analogique avec un temps d'intégration de $10 \mathrm{~s}$ (NPLC 500). Le boîtier, contenant le MEMS et l'électronique de commande, est équipé de capteurs de température $(T)$, d'humidité relative $(H R)$ et de pression $(P)$. Ces paramètres sont enregistrés simultanément avec les mesures de stabilité de la tension du MEMS. 


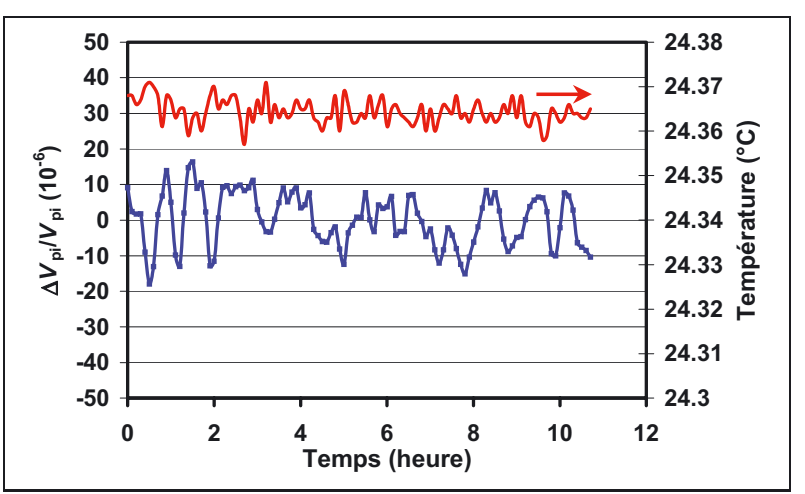

Fig. 26. - Stabilité de la tension de pull-in du MEMS 5V-HK6 à $100 \mathrm{kHz}$.

La figure 26 présente l'écart relatif par rapport à la moyenne de la tension $V_{\text {pi }}$ du MEMS 5V-HK6 (tension de pull-in de $4 \mathrm{~V}$ ) mesurée à $100 \mathrm{kHz}$ durant $12 \mathrm{~h}$ et sous les conditions suivantes :

$$
\begin{aligned}
& 24,36{ }^{\circ} \mathrm{C}<T<24,37{ }^{\circ} \mathrm{C} ; \\
& 43,2 \%<H R<44,4 \% \\
& 995 \mathrm{hPa}<P<999 \mathrm{hPa} .
\end{aligned}
$$

L'écart type ( $1 \sigma)$ sur la moyenne de cette série de mesures de la tension de pull-in est de l'ordre de $6,3 \times 10^{-6}$, ce qui constitue à cette fréquence de $100 \mathrm{kHz}$, un très bon résultat dans le domaine des tensions alternatives. Pour d'autres échantillons, cet écart type peut atteindre $30 \times 10^{-6}$. Plusieurs raisons peuvent être avancées pour expliquer ce comportement :

- l'épaisseur de $60 \mu \mathrm{m}$ de la couche active de silicium du substrat SOI impose, pour avoir des tensions de pull-in inférieures à $10 \mathrm{~V}$, des dimensions des ressorts où la largeur est typiquement de $10 \mu \mathrm{m}$. Cette configuration est très défavorable pour un déplacement vertical de la membrane mobile avec une flexion des ressorts dans cette direction. Le profil des ressorts $(60 \mu \mathrm{m} \times 10 \mu \mathrm{m})$ impose un axe préférentiel de déplacement et de contrainte qui n'est pas sollicité ici par le mouvement vertical de l'électrode mobile;

- les électrodes ne sont pas métallisées et, même si le silicium est fortement dopé, un oxyde naturel peut se former sur la surface. Des charges électriques d'espace peuvent alors s'accumuler et ce phénomène peut causer ces instabilités de la tension;

- les composants MEMS sont collés sur le support TO8 à l'aide d'une résine époxy conductrice dont les caractéristiques électriques et la tenue mécanique sont loin d'être optimales. En effet, un des contacts électriques de la capacité MEMS passe par cette colle puisque le substrat n'est autre que l'une des électrodes. Une résistance électrique vient donc se mettre en série avec la capacité des MEMS et ses variations impacteront directement la tension de sortie de l'amplificateur de contre-réaction.

\section{Conclusion}

Cet article présente les premiers résultats obtenus sur le développement de références de tension à base de MEMS. Des structures de test ont été d'abord conçues avec un logiciel par éléments finis. Plusieurs prototypes MEMS à membrane circulaire en silicium ont été réalisés avec des ressorts de différentes géométries et dimensions ; ils permettent un déplacement vertical. L'objectif de cette première réalisation est, d'une part, d'évaluer les performances des outils de calculs et de simulation pour concevoir des architectures spécifiques et, d'autre part, de tester les capacités d'un procédé industriel SOI pour la fabrication de ces structures MEMS. Ces dispositifs MEMS ont été réalisés avec le procédé industriel MPW (Multiproject Wafer) de la société Tronics microsystems à partir d'un substrat SOI. Un emballage hermétique sous vide ou non hermétique a été utilisé pour protéger les MEMS. Les tensions de pull-in mesurées sur ces échantillons varient de $2 \mathrm{~V}$ à $100 \mathrm{~V}$ en parfait accord avec les simulations. Les résultats obtenus sur ces dispositifs de test ont permis de concevoir une deuxième génération de microsystèmes avec des caractéristiques améliorées.

L'électronique de commande basée sur la modulation d'amplitude (AM) a été également développée. Elle a été spécifiquement adaptée aux caractéristiques des MEMS en terme de capacité nominale $C_{0}$ et de tension de pull$i n$. La dernière génération du montage électronique de commande intègre également une régulation de la température du dispositif MEMS lui-même par des cellules Peltier.

Les mesures des tensions de pull-in des MEMS (5 V et $10 \mathrm{~V}$ ) à membrane circulaire réalisées à $100 \mathrm{kHz}$ montrent une très bonne stabilité de la tension de référence avec un écart type de l'ordre de $6,3 \times 10^{-6}$ sur 12 h. Ce niveau de stabilité de la tension de pull-in intègre non seulement celle des MEMS, mais aussi celle de l'électronique de commande et du multimètre de mesure. Ce résultat est d'ores et déjà remarquable comparé aux performances des convertisseurs AC-DC à ces fréquences. Cependant, d'autres échantillons ont montré une stabilité moindre avec des écarts types pouvant atteindre $30 \times 10^{-6}$. La raison principale vient certainement du SOI utilisé dans le procédé Tronics qui privilégie des déplacements dans le plan du substrat. Il est donc nécessaire de développer un procédé technologique utilisant des substrats SOI adaptés pour le déplacement vertical. De nouveaux dispositifs MEMS à peignes silicium interdigités sont également développés.

\section{Références}

[1] VAN DRIEËNHUIZEN B.P., "Integrated Electrostatic RMS to Converter", Thèse de doctorat, Univ. de Delft, PaysBas, 1996.

[2] VAN DRIEËNHUIZEN B.P. et WOLFFENBUTTEL R.F., "Integrated Micromachined Electrostatic True RMS-toDC Converter", IEEE Trans. Instrum. Meas., 44, 2, 1995, 370-373. 
[3] Suhonen M., SEPPÄ H., OJA A.S., HeInILÄ M. et NÄKKI I., "AC and DC Voltage Standards Based on Silicon Micromechanics, in Digest", CPEM98, Washington DC, Etats-Unis d'Am., juillet 1998, 23-24.

[4] KärkKäinen A., Pesonen N., Suhonen M., OJA A., MANNINEN A., TISNEK N. et SEPPÄ H., "MEMS based AC Voltage Reference", IEEE Trans. Instrum. Meas., 54, 2005, 595-599.

[5] KÄRKKÄINEN A., AWAN S.A., KYYNÄRÄINEN J., PEKKo P., OJA A. et SEPPÄ H., "Optimized Design and Process for Making a DC Voltage Reference Based on MEMS", IEEE Trans. Instrum. Meas., 54, 2005, 563-566.

[6] Cretu E., Rocha L.A. et WolfFenbuttel R.F., "Micromechanical Voltage reference Using the Pull-In of a Beam", IEEE Trans. Instrum. Meas., 50, 2001, 15041507.

[7] KYYNÄRÄINEN J., OJA A.S. et SEPPÄ H., "A Micromechanical RMS-to-DC Converter, in Digest", CPEM2000, Sydney, Australie, mai 2000, 699-700.

[8] de Graaf G., Bartek M., Xiao Z., van Mullem C.J. et WolfFEnButTEL R.F., "Bulk Micromachined Electrostatic True RMS-to-DC Converter", IEEE Trans. Instrum. Meas., 50, 6, 2001, 1508-1512.

[9] SEPPÄ H., KYYNÄRÄINEN J. et OJA A., "Microelectromechanical Systems in Electrical Metrology", IEEE Trans. Instrum. Meas., 50, 2, 2001, 440-444.

[10] KYYNÄRÄINEN J., OJA A.S. et SEPPÄ H., "Stability of Microelectromechanical Devices for Electrical Metrology”, IEEE Trans. Instrum. Meas., 50, 6, 2001, 1499-1503.

[11] Fernandez L.J., VisSer E., Sesé J., Wiegerink R.J., JANSEN H.V., Flokstra J. et ElWenspoeK M., "Development of a capacitive MEMS RF power sensor without dissipative losses: towards a new philosophy of RF power sensing", CPEM04, Londres, Roy.-Uni, 27 juin-2 juillet, 2004

[12] Fernandez L.J., VisSer E., Sesé J., Wiegerink R.J., Jansen H.V., FloKstra J. et ElWenspoek M., "Capacitive rf power sensor based on MEMS technology", IEEE Sensors Conference, Toronto, Canada, 22-24 octobre, 2003.

Article reçu le 25 mars 2010, version révisée reçue le 30 août 2010.
[13] Alastalo A., KyYnÄrÄINEN J., SEPPÄ H., KÄRKKÄINEN A., PESONEN N., LAHDES M., VÄH ̈̈-HeIKKILÄ T., PEKKo P. et DEKKER J., "Wideband microwave power sensor Based on MEMS technology", CPEM04, Londres, Roy.-Uni, 27 juin-2 juillet, 2004.

[14] CHU P.B. et PISTER K.S.J., "Analysis of closed-loop control of parallel-plate electrostatic microgrippers", in Proc. IEEE Int. Conf. Robotics and Automation, San Diego, Californie, Etats-Unis d'Am., mai 1994, 820-5.

[15] SeEger J.I. et Crary S.B., "Stabilization of electrostatically actuated mechanical devices", in Proc. Transducers'97, Chicago, Etats-Unis d'Am., IL, juin 1997, 1133-1136.

[16] Chan E.K. et DutTon R.W., "Electrostatic micromechanical actuator with extended range of travel", $J$. Microelectromechan. Syst., 9, 2000, 321-328.

[17] SeEger J.I. et Boser B.E., "Charge Control of ParallelPlate, Electrostatic Actuators and the Tip-In Instability", J. Microelectromechan. Syst., 12, 5, 2003, 116.

[18] SEEger J.I. et Boser B.E., "Dynamics and control of parallel-plate actuators beyond the electrostatic instability", in Proc. 10th Int. Conf. On Solid-state Sensors and Actuators, Sendai, Japon, juin 7-10, 1999.

[19] SeEger J.I. et Boser B.E., "Charge Control of ParallelPlate, Electrostatic Actuators and the Tip-In Instability", J. Microelectromechan. Syst., 12, 5, 2003, 1-16.

[20] Nadal-Guardia R., Dehé A., Aigner R. et CASTAÑER L.M., "Current Drive Methods to Extend the Range of Travel of Electrostatic Microactuators Beyond the Voltage PullIn Point", J. Microelectromechan. Syst., 11, 3, 2002, 255-263.

[21] "EMMA project report", Information Societies Technology, IST200028261EMMA, 2005.

[22] KÄRKK ÄINEN A., Tisnek N., MANNINEN A., PESONEN N., OJA A. et SEPPÄ H., "Electrical stability of a MEMSbased AC voltage reference", Sensors $\mathcal{E}$ Actuators A, 137, 2007, 169-174.

[23] Camon H. et Ganibal C., "Advantages of alternative actuating signal for MEMS actuators", Sensors $\mathcal{E}$ Actuators A, 136, 1, 2007, 299-303. 